\section{A bitter taste for sinus infections}

\section{By Lauren Martz, Staff Writer}

Modulating taste receptors in upper airways might provide a new way to treat chronic rhinosinusitis, according to a study from the University of Pennsylvania that shows bitter taste agonists stimulate the release of antimicrobial peptides in the sinuses. ${ }^{1}$ Taste receptortargeted therapies could reduce patient dependence on antibiotics, but pinpointing the right taste receptor for an individual's infection might be the biggest challenge.

The UPenn team is now optimizing delivery of taste receptor ligands to the nasal cavity for a planned clinical study in rhinosinusitis and is developing a taste test-based diagnostic to link receptor polymorphisms to specific infections.

Antibiotics combined with steroids are standard treatment in chronic rhinosinusitis, a disease involving persistent infection or inflammation of the nasal sinus cavities. However, the drugs fail to control the infection in a substantial number of patients, and antibiotic overuse contributes to the emergence of resistant bacterial strains.

In patients who do not respond to standard therapy, surgery is used to open the nasal passages. However, some patients also fail to respond to surgery.

To find alternatives to antibiotics, researchers are trying to harness the innate immune system to overcome airway infections. Recently, bitter taste receptors in airway epithelia have emerged as important players in the innate host defense. ${ }^{2}$

In 2012, Noam Cohen and his team found that Gram-negative bacteria in the upper airways can stimulate the bitter taste receptor type 2 member 38 (TAS2R38), thus triggering secretion of bactericidal levels of nitric oxide. ${ }^{3}$

His team also found that patients with an inactivating TAS2R38 polymorphism had an increased risk of developing chronic sinusitis from Pseudomonas aeruginosa and other Gram-negative infections. ${ }^{3}$

Now, Cohen and colleagues have identified a pathway linking taste receptor stimulation to bactericidal activity and have discovered effects of bitter and sweet taste receptors that could be exploited therapeutically.

Cohen is an associate professor of otorhinolaryngology: head and neck surgery at the Philadelphia VA Medical Center and director of rhinology research and chair of the Otorhinolaryngology Resident Research Community at the Perelman School of Medicine at the University of Pennsylvania. The team also included researchers from The Children's Hospital of Philadelphia and Monell Chemical Senses Center.

\section{Bitter receptors, better defense}

To test how taste receptor agonists influence innate immunity, the team used primary human sinonasal cells cultured at an air-liquid interface, which mimics the nasal epithelium in the sinuses. Fluid collected at the interface represents the equivalent of sinonasal secretions.

The team tested agonists of eight bitter taste receptors-not including TAS2R38 - to find out whether other taste receptors can also modify antimicrobial activity.

The bitter taste agonists denatonium benzoate and absinthin elicited secretions that produced potent bactericidal activity against P. aeruginosa, methicillin-resistant Staphylococcus aureus (MRSA), Klebsiella pneumoniae and S. epidermis. Denatonium benzoate and absinthin are commercially available as chemicals.

The team then studied the composition of the antimicrobial secretions and found that $\beta$-defensins, small antimicrobial peptides, were upregulated by the bitter taste agonists. Antibodies against $\beta$-defensins decreased the bactericidal effects of the secretions against $P$. aeruginosa and MRSA compared with no antibodies but did not cause complete inhibition.

Cohen's team concluded that $\beta$-defensins represent a significant part of the antibacterial mechanism triggered by bitter taste receptors, although other antimicrobial peptides are likely involved too. Also, because the $\beta$-defensin response was absent in bronchial cells, the team proposed the effect to be specific to upper airway innate immunity.

Finally, nasal secretions from patients with chronic rhinosinusitis generally exhibit higher glucose levels than secretions from healthy controls.

Because the cells expressing the bitter taste receptors also express sweet taste receptors in the nasal sinuses, the researchers tested the effect of sugars in their in vitro model, showing that glucose and other sweet taste receptor agonists inhibited the antimicrobial effects of the culture secretions. Cohen and colleagues thus inferred that sweet receptor activation blocks the effects of bitter taste receptors.

Based on these data, the team proposed a model in which damage to the epithelial barrier caused by chronic infection allows glucose leakage into the nasal mucosa. The increased mucosal glucose concentration then suppresses the antimicrobial effects of bitter receptors and impairs a patient's innate ability to clear the infection.

Data were published in The Journal of Clinical Investigation.

Cohen told SciBX that his team plans to pursue therapeutic development based on this work and is considering bitter taste receptor agonists and sweet taste receptor antagonists.

\section{Delivering taste to the nose}

According to Lisa Earnhardt, the antibacterial activity of taste receptors could present a major step forward in rhinosinusitis.

"This connection between bitter and sweet taste receptors to chronic sinusitis offers an entirely new avenue to diagnose and treat upper airway conditions," she said. "Activating the innate immune response by stimulating the native sinonasal taste receptors has the potential to drastically decrease the use of antibiotics in the management of upper respiratory infections."

Earnhardt is president and CEO of Intersect ENT Inc. The company has a drug-eluting stent approved for treatment of sinusitis. 
"This work represents a paradigm shift for how sinusitis is managed," said Martin Desrosiers. "In addition to the millions of patients who suffer from sinusitis, there are 500,000 to 1 million sinusitis patients who do not respond to any steroids or antibiotics. This is actually a sizeable market, and the severity and chronic nature of the disease help justify the expense of new therapeutic development."

Desrosiers is a clinical professor and program director of otolaryngology-head and neck surgery at the University of Montreal.

Cohen said that nasal irrigation is his first choice as a delivery method for chronic sinusitis because it targets the source of the problem and is likely to produce low systemic exposure, but mucosal absorption is something that might need to be investigated. $\mathrm{He}$ added that because bitter taste receptors are being found all over the body, however, the possible effects of any absorption are hard to predict.

Vijay Ramakrishnan said that the study of airway taste receptors is still in its infancy. "Modulation of this system may have a number of unforeseen downstream effects, and the interactions with other immune defense mechanisms are just now being explored."

He added that preclinical models might be of limited predictive value because animal models of upper respiratory infections do not closely mimic the human system.

Ramakrishnan is an assistant professor of rhinology and co-director of the Skull Base Program at the University of Colorado Denver School of Medicine.

Cohen said that his group will look for alterations in thyroid function-a possible side effect suggested by the similarity of another TAS2R38 agonist, propylthiouracil (PTU), used to treat goiter. He added that impaired olfactory function is also something that should be considered with any intranasal medications.

Cohen told SciBX that it is unclear what the regulatory path for these compounds might be. "At this point, we are unsure about how the FDA will look at the taste receptor modulators. These are tested substances, but they will be administered through a new route," he said.

Sweet taste receptor antagonists such as lactisole are already available on the market as food additives. Bitter taste receptor agonists are used as aversive agents to prevent accidental ingestion of various substances.

According to Desrosiers, there are no therapeutics specifically approved for chronic rhinosinusitis on the market. "It was only recently that the FDA even recognized, defined and offered guidance on chronic rhinosinusitis. It is mostly guesswork using a variety of offlabel antibiotics and steroids," he said.

Cohen told SciBX that although the group has tested food additives and aversive agents, it is also looking for new bitter taste agonists and sweet taste antagonists with higher affinities than the available compounds. The compounds being considered for clinical testing are undisclosed.

\section{Personal taste}

One major challenge is finding the right agent to use in any individual because of the variety of pathogens that can cause rhinosinusitis and the genetic variability of taste receptors.

"There is a huge uphill battle for clinical trials aimed at providing efficacy in the indication because this is not a single disease caused by a single agent," Cohen said.

"We know that there are multiple bitter taste receptors that each respond to different types of pathogens," he said. "For example, TAS2R38 responds to Gram-negative bacteria, and data suggest that there may be other receptors that exist for Gram-positive bacteria. Each of these receptors has complex genetics, and it is possible that if you have a mutation in one, you could be susceptible to a certain type of infection."

Daniel Hamilos, an associate professor of medicine at Massachusetts General Hospital, said that because the team showed that problems in bitter taste receptor signaling are associated with impaired sinonasal immunity, the team will next need to determine whether taste receptor modulation is effective at restoring receptor-mediated immunity in patients with receptor mutations.

According to Thomas Finger, it may be possible to design a therapeutic that stimulates the pathway downstream of the taste receptor to bypass problems of receptor variability. Finger is a professor of cell and developmental biology at the University of Colorado Denver School of Medicine.

Cohen told SciBX that his team is working on a diagnostic test using taste testing to identify pathogens responsible for chronic rhinosinusitis in individual patients.

"We think taste receptor function in the nose may mimic that of the tongue, and it is possible to test for specific taste receptor stimulation on the tongue. This could be developed as a comprehensive diagnostic to help identify certain individual susceptibilities. An inability to taste one bitter receptor agonist could help indicate susceptibility to chronic infections by a particular bacterial or fungal species," he said.

Ramakrishnan said that the next steps for diagnostic development should be to develop a more complete understanding of the different taste receptors in the human airway epithelium, establish what pathogenic species the receptors can detect and identify the genetic and acquired defects in the receptor pathways.

According to Cohen, the diagnostic potential could go beyond identifying the specific pathogenic species that cause disease in patients.

"This type of diagnostic may also help predict whether patients would benefit from sinus surgery. Some patients don't respond well to surgery and some actually get worse. We've found that it may be possible to predict whether patients will benefit from surgery based on receptor genotype." 


\section{ANALYSIS}

Cohen said that the UPenn team has filed a patent application covering the work. The IP is available for licensing.

Martz, L. SciBX 7(12); doi:10.1038/scibx.2014.331

Published online March 27, 2014

\section{REFERENCES}

1. Lee, R.J. et al. J. Clin. Invest.; published online Feb. 17, 2014; doi:10.1172/JCl72094

Contact: Noam A. Cohen, Perelman School of Medicine at the University of Pennsylvania, Philadelphia, Pa. e-mail: cohenn@uphs.upenn.edu

2. Lee, R.J. \& Cohen, N.A. Am. J. Rhinol. Allergy 27, 283-286 (2013)
3. Lee, R.J. et al. J. Clin. Invest. 122, 4145-4159 (2012)

COMPANIES AND INSTITUTIONS MENTIONED

The Children's Hospital of Philadelphia, Philadelphia, $\mathrm{Pa}$. Intersect ENT Inc., Palo Alto, Calif.

Massachusetts General Hospital, Boston, Mass.

Monell Chemical Senses Center, Philadelphia, Pa.

Perelman School of Medicine at the University of Pennsylvania, Philadelphia, Pa.

Philadelphia VA Medical Center, Philadelphia, $\mathrm{Pa}$.

University of Colorado Denver School of Medicine, Aurora, Colo.

University of Montreal, Montreal, Quebec, Canada

University of Pennsylvania, Philadelphia, $\mathrm{Pa}$. 